\title{
Robust Synchronization Control of Uncertain Fractional-Order Chaotic Systems via Disturbance Observer
}

\author{
Kaijuan Xue and Yongbing Huangfu \\ Department of Mechanical and Electronic Engineering, Shanxi Engineering Vocational College, Taiyuan 030009, China \\ Correspondence should be addressed to Yongbing Huangfu; hf200133w@163.com
}

Received 23 August 2021; Revised 16 September 2021; Accepted 17 September 2021; Published 4 October 2021

Academic Editor: Shenggang Li

Copyright ( $\odot 2021$ Kaijuan Xue and Yongbing Huangfu. This is an open access article distributed under the Creative Commons Attribution License, which permits unrestricted use, distribution, and reproduction in any medium, provided the original work is properly cited.

\begin{abstract}
This paper studies the synchronization of two different fractional-order chaotic systems through the fractional-order control method, which can ensure that the synchronization error converges to a sufficiently small compact set. Afterwards, the disturbance observer of the synchronization control scheme based on adaptive parameters is designed to predict unknown disturbances. The Lyapunov function method is used to verify the appropriateness of the disturbance observer design and the convergence of the synchronization error, and then the feasibility of the control scheme is obtained. Finally, our simulation studies verify and clarify the proposed method.
\end{abstract}

\section{Introduction}

Fractional calculus has a history of more than 300 years. It has been shown that most of the physical phenomena in nature exist in the form of fractional order, and furthermore, the conventional integer-order calculus is only a special case of the fractional calculus $[1,2]$. Compared with integer calculus, the fractional calculus model is closer to the real world and has broad prospects for development. Fractional calculus has been proven to be a valuable tool for modeling many physical phenomena such as system biology, physics, chemistry, automation, and so on [3-7]. Recently, many authors have begun to study the chaotic dynamics of fractional dynamical systems [8]. As we all know, chaotic synchronization has important theoretical significance and practical value and has made great contributions due to its potential applications in many scientific and engineering fields $[9,10]$. Various chaotic synchronization systems have been proposed, and researchers have obtained many theoretical results due to their quite powerful attraction, including complete synchronization [11], partial synchronization [12], antisynchronization [13], generalized synchronization [14], projection synchronization [15], time scale synchronization, composite synchronization [16], and so on.
In the process of chaos synchronization, it is very hard to tackle uncertainties and unknown disturbances, and if systems uncertainties are not considered, it will damage the applications of the chaos synchronization. In fact, the unknown parameters and disturbances exist in most of realworld systems. In this paper, a kind of drive response synchronization scheme is considered, and the response system is designed with unknown disturbances to be monitored by the disturbance observer [17], and the control scheme of adaptive parameters [18] is used to synchronize the two systems.

In some related works, a robust technique, i.e., disturbance observer, is not only used to approximate external disturbances but also as an effective robust control method because the influence of system uncertainties can be estimated and compensated based on the output of the disturbance observer. In [19], a fuzzy disturbance observer for discrete-time systems and its applications were studied. In [20], a nonlinear predictive control was proposed in combination with a disturbance observer. The general framework of disturbance observer-based control for a disturbed nonlinear system was presented in [21]. Then, a kind of disturbance observer will be designed to monitor unknown disturbances. For the problem of mismatch disturbance, 
traditional methods are difficult to deal with the control problem, so the paper proposes an adaptive parameter control method based on a disturbance observer to deal with the problem of mismatch disturbance. The new method combines disturbance estimation with the controller design. It can solve the problem better.

Inspired by the abovementioned works, this paper applies a synchronization method that is different from the previous synchronization methods. The proposed synchronization technology is based on the idea of tracking control and stability theory of fractional-order systems, which is simple and theoretically rigorous. The method derived in this paper shows that the synchronization between different fractional-order chaotic systems can be achieved. Compared with the existing results, the main work of this paper is summarized as follows. (1) A feasible synchronization controller is proposed for fractional-order chaotic systems with system uncertainties. (2) A type of disturbance observer is designed to effectively monitor unknown disturbances.

The structure of this article is as follows. Section 2 introduces the preliminaries and a class of three-dimensional chaotic systems. The design of disturbance observers and controllers are given to ensure the stability of the system in Section 3. The data simulation results are shown to verify the feasibility of the control scheme in Section 4. Finally, Section 5 presents the conclusions of this paper.

\section{Preliminaries and Problem Statement}

There are many definitions of fractional derivatives, but the Caputo fractional-order derivative definition is one of the most widely utilized and can be used in most engineering applications because it contains the initial conditions of the original function and the integer-order derivative. Hence, we will employ the Caputo fractional derivative, and the lower bound of the integral of fractional calculus is set to 0 in this article.

Definition 1 (see [22]). The Caputo fractional derivative with order $v$ can be expressed as

$$
\mathscr{D}_{t}^{\nu} f(t)=\frac{1}{\Gamma(n-\nu)} \int_{t_{0}}^{t}(t-\tau)^{\nu+n-1} f^{(n)}(\tau) \mathrm{d} \tau,
$$

where $v$ represents any real number, $n$ denotes an integer constant and $n-1 \leq \nu<\nu, n=[\nu]+1$, and the $\Gamma(\cdot)$ is the Euler gamma function, which is defined as $\Gamma(n-v)$ $=\int_{0}^{\infty} t^{n-\nu-1} e^{-t} \mathrm{~d} t$.

We are expected to introduce some important properties of fractional derivatives as follows.

Property 1 (see [23]). If $f(t)$ is a constant function, then

$$
\mathscr{D}_{t}^{v} f(t)=0 .
$$

Property 2 (see [24]). If the fractional derivatives of functions $f_{1}(t)$ and $f_{2}(t)$ exist, the linearity of Caputo fractional derivative can be expressed as

$$
\mathscr{D}_{t}^{\nu}\left[\varrho_{1} f_{1}(t)+\varrho_{2} f_{2}(t)\right]=\varrho_{1} \mathscr{D}_{t}^{\nu} f_{1}(t)+\varrho_{2} \mathscr{D}_{t}^{\nu} f_{2}(t),
$$

where $\varrho_{1}$ and $\varrho_{2}$ are two constants.

Lemma 1 (see [25]). Let $f(t) \in \mathbb{R}$ is a continuous and differentiable function. Then, for any time instant $t>t_{0}$, the following inequality holds:

$$
\mathscr{D}_{t}^{v} f^{2}(t) \leq 2 f(t) \mathscr{D}_{t}^{v} f(t) .
$$

Remark 1. If $f(t) \in \mathbb{R}^{n}$ is a smooth function, we can get

$$
\mathscr{D}_{t}^{v}\left(f^{T}(t) f(t)\right) \leq 2 f^{T}(t) \mathscr{D}_{t}^{\nu} f(t) .
$$

Lemma 2. Suppose $V(t)$ is continuous and differentiable and satisfies that

$$
\mathscr{D}_{t}^{\nu} V(t) \leq-A_{1} V(t)+A_{2},
$$

where $A_{1}, A_{2}>0$ are constants, then there exists a constant $t_{0}$ such that

$$
|V(t)| \leq \frac{2 A_{2}}{A_{1}},
$$

for $t>t_{0}$.

The control of fractional-order nonlinear dynamic systems is an important research subject, but it is rarely studied in the presence of interference. Consider a class of fractionalorder chaotic systems that are expressed as

$$
\left\{\begin{array}{l}
\mathscr{D}_{t}^{v} x_{1}(t)=a x_{1}(t)+b x_{2}(t) x_{3}(t) \\
\mathscr{D}_{t}^{v} x_{2}(t)=c x_{2}(t)+x_{1}(t) x_{3}(t) \\
\mathscr{D}_{t}^{v} x_{3}(t)=d x_{3}(t)+e x_{1}(t) x_{2}(t)+x_{2}^{2}(t),
\end{array}\right.
$$

where $a, b, c, d$, and $e$ are system parameters and $x(t)=$ $\left[x_{1}(t), x_{2}(t), x_{3}(t)\right]^{T}$ are the state variables of the master system.

\section{Controller Design and Stability Analysis}

This section is based on the disturbance observer to observe the unknown disturbance and adopts the adaptive control method to synchronize system (8) with system (9).

3.1. Design of Disturbance Observer. In this section, the unknown disturbances are observed based on the disturbance observer, and the adaptive control method is adopted to ensure that the system is in a stable state for the fractionalorder nonlinear system.

According to system (8), its subordinate system is given 


$$
\left\{\begin{array}{l}
\mathscr{D}_{t}^{\nu} \xi_{1}(t)=a \xi_{1}(t)+b \xi_{2}(t) \xi_{3}(t)+\delta_{1}(t)+u_{1}(t) \\
\mathscr{D}_{t}^{v} \xi_{2}(t)=c \xi_{2}(t)+\xi_{1}(t) \xi_{3}(t)+\delta_{2}(t)+u_{2}(t) \\
\mathscr{D}_{t}^{v} \xi_{3}(t)=d \xi_{3}(t)+e \xi_{1}(t) \xi_{2}(t)+\xi_{2}^{2}+\delta_{3}(t)+u_{3}(t)
\end{array}\right.
$$

where $\delta(t)=\left[\delta_{1}(t), \delta_{2}(t), \delta_{3}(t)\right]^{T}$ corresponds to unknown external disturbance and $u(t)=\left[u_{1}(t), u_{2}(t), u_{3}(t)\right]^{T}$ represents the control input of the system.

Assumption 1. The external disturbance $\delta_{i}(t)(i=1,2,3)$ are continuous, and Caputo fractional of $\delta_{i}$ is bounded with an unknown upper bound, i.e., $\left|\mathscr{D}_{t}^{v} \delta_{i}(t)\right| \leq \bar{\delta}_{i}\left(\left\|\mathscr{D}_{t}^{v} \delta(t)\right\| \leq \bar{\delta}\right)$, for arbitrary $t>0$ where $\bar{\delta}$ is an unknown constant.

In the following, a fractional-order disturbance observer is proposed to approximate the unknown disturbance so that the subordinate system can track the master system to achieve synchronization because the external disturbance described above is completely fuzzy and unknown on the basis of the controller. To facilitate the design of the disturbance observer, the following auxiliary variables are involved:

$$
\chi_{i}(t)=\delta_{i}(t)-\rho_{i} \xi_{i}(t)
$$

where $\rho_{i}(i=1,2,3)$ is a positive constant and $\chi(t)=\left[\chi_{1}(t), \chi_{2}(t), \chi_{3}(t)\right]^{T}$. Taking (10) into account, the fractional time derivative of the $\chi_{i}(t)$ can be written as

$$
\mathscr{D}_{t}^{v} \chi_{i}(t)=\mathscr{D}_{t}^{v} \delta_{i}(t)-\rho_{i} \mathscr{D}_{t}^{v} \xi_{i}(t)
$$

Substituting (9) into (11), it brings about that

$$
\left\{\begin{array}{l}
\mathscr{D}_{t}^{v} \chi_{1}(t)=\mathscr{D}_{t}^{v} \delta_{1}(t)-\rho_{1}\left[a \xi_{1}(t)+b \xi_{2}(t) \xi_{3}(t)+\delta_{1}(t)+u_{1}(t)\right] \\
\mathscr{D}_{t}^{v} \chi_{2}(t)=\mathscr{D}_{t}^{v} \delta_{2}(t)-\rho_{2}\left[c \xi_{2}(t)+\xi_{1}(t) \xi_{3}(t)+\delta_{2}(t)+u_{2}(t)\right] \\
\mathscr{D}_{t}^{v} \chi_{3}(t)=\mathscr{D}_{t}^{v} \delta_{3}(t)-\rho_{3}\left[d \xi_{3}(t)+e \xi_{1}(t) \xi_{2}(t)+\xi_{2}^{2}+\delta_{3}(t)+u_{3}(t)\right] .
\end{array}\right.
$$

Thus, combining (10) and (12), we have

$$
\left\{\begin{array}{l}
\mathscr{D}_{t}^{v} \chi_{1}(t)=\mathscr{D}_{t}^{v} \delta_{1}(t)-\rho_{1}\left[a \xi_{1}(t)+b \xi_{2}(t) \xi_{3}(t)+\chi_{1}(t)+\rho_{1} \xi_{1}(t)+u_{1}(t)\right], \\
\mathscr{D}_{t}^{v} \chi_{2}(t)=\mathscr{D}_{t}^{v} \delta_{2}(t)-\rho_{2}\left[c \xi_{2}(t)+\xi_{1}(t) \xi_{3}(t)+\chi_{2}(t)+\rho_{2} \xi_{2}(t)+u_{2}(t)\right], \\
\mathscr{D}_{t}^{v} \chi_{3}(t)=\mathscr{D}_{t}^{v} \delta_{3}(t)-\rho_{3}\left[d \xi_{3}(t)+e \xi_{1}(t) \xi_{2}(t)+\xi_{2}^{2}+\chi_{3}(t)+\rho_{3} \xi_{3}(t)+u_{3}(t)\right] .
\end{array}\right.
$$

According to (10), the disturbance can be estimated as

$$
\widehat{\delta}_{i}(t)=\widehat{\chi}_{i}(t)+\rho_{i} \xi_{i}(t) .
$$

To calculate the disturbance estimate, the estimations of the intermediate auxiliary variables are expressed as

$$
\left\{\begin{array}{l}
\mathscr{D}_{t}^{v} \widehat{\chi}_{1}(t)=-\rho_{1}\left[a \xi_{1}(t)+b \xi_{2}(t) \xi_{3}(t)+\rho_{1} \xi_{1}(t)\right]-\rho_{1} \widehat{\chi}_{1}(t)-\rho_{1} u_{1}(t), \\
\mathscr{D}_{t}^{v} \widehat{\chi}_{2}(t)=-\rho_{2}\left[c \xi_{2}(t)+\xi_{1}(t) \xi_{3}(t)+\rho_{2} \xi_{2}(t)\right]-\rho_{2} \widehat{\chi}_{2}(t)-\rho_{2} u_{2}(t), \\
\mathscr{D}_{t}^{v} \widehat{\chi}_{3}(t)=-\rho_{3}\left[d \xi_{3}(t)+e \xi_{1}(t) \xi_{2}(t)+\xi_{2}^{2}+\rho_{3} \xi_{3}(t)\right]-\rho_{3} \widehat{\chi}_{3}(t)-\rho_{3} u_{3}(t),
\end{array}\right.
$$

where $\hat{\chi}_{i}(t)$ is an estimation of $\chi_{i}(t)$. Using (10) and (14), the following result is deduced:

$$
\tilde{\chi}_{i}(t)=\widetilde{\delta}_{i}(t)
$$

where $\widetilde{\chi}_{i}(t)=\chi_{i}(t)-\widehat{\chi}_{i}(t), \widetilde{\delta}_{i}(t)=\delta_{i}(t)-\widehat{\delta}_{i}(t)$, and $\widetilde{\delta}(t)=$ $\left[\widetilde{\delta}_{1}(t), \widetilde{\delta}_{2}(t), \widetilde{\delta}_{3}(t)\right]^{T}$.

To take the fractional derivative of (16), and considering (13) and (15), we obtain

$$
\mathscr{D}_{t}^{\nu} \widetilde{\chi}_{i}(t)=\mathscr{D}_{t}^{\nu} \delta_{i}(t)-\rho_{i} \widetilde{\chi}_{i}(t) .
$$

The detailed design principle of the disturbance observer is summarized in the following theorem.

Theorem 1. Consider the fractional-order chaotic system (8) and the subordinate system (9) with unknown disturbance. The disturbance error is obtained by using the disturbance observers (14) and (15), and then the disturbance approximation error $\widetilde{\delta}$ will converge to an arbitrary small compact set $\Omega_{\delta}$ defined by 


$$
\Omega_{\tilde{\delta}}:=\left\{\widetilde{\delta} \in R^{3} \mid\|\widetilde{\delta}\| \leq \sqrt{\frac{4 B}{B_{0}}}\right\},
$$

where $B_{0}$ and $B$ will be given later.

Proof. In order to verify the practicality of the above disturbance observer, we choose the following Lyapunov function to express the stability analysis of the disturbance error:

$$
V_{\delta}=\frac{1}{2} \widetilde{\delta}^{T}(t) \widetilde{\delta}(t)=\frac{1}{2} \widetilde{\chi}^{T}(t) \widetilde{\chi}(t) .
$$

Based on Lemma 1 and Assumption 1, the Caputo derivative of the Lyapunov function (19) is written as

$$
\begin{aligned}
V_{\delta} & \leq \widetilde{\delta}^{T}(t) \mathscr{D}_{t}^{\nu} \widetilde{\delta}(t)=\widetilde{\chi}^{T}(t) \mathscr{D}_{t}^{\nu} \widetilde{\chi}(t)=-\widetilde{\chi}^{T}(t) \rho \widetilde{\chi}(t)+\widetilde{\chi}^{T}(t) \mathscr{D}_{t}^{\nu} \delta(t) \\
& \leq-\widetilde{\chi}^{T}(t) \rho \widetilde{\chi}(t)+\frac{1}{2}\left\|\tilde{\chi}^{T}(t)\right\|^{2}+\frac{1}{2}\left\|\mathscr{D}_{t}^{\nu} \delta(t)\right\|^{2} \\
& \leq-\tilde{\chi}^{T}(t)\left(\rho-0.5 I_{3 \times 3}\right) \widetilde{\chi}(t)+\frac{1}{2} \bar{\delta}^{2}(t) \\
& =-\widetilde{\delta}^{T}(t)\left(\rho-0.5 I_{3 \times 3}\right) \tilde{\delta}(t)+\frac{1}{2} \bar{\delta}^{2}(t) \leq-B_{0} V_{\delta}(t)+B,
\end{aligned}
$$

where $\rho=\operatorname{diag}\left(\rho_{1}, \rho_{2}, \rho_{3}\right)$ and $B_{0}$ and $B$ satisfy

$$
\begin{aligned}
B_{0} & =2 \lambda_{\min }\left(\rho-0.5 I_{3 \times 3}\right), \\
B & =\frac{1}{2} \bar{\delta}^{2} .
\end{aligned}
$$

To ensure the boundedness of the closed-loop system, the matrix $\rho$ designed by the disturbance observer should satisfy $\rho-0.5 I_{3 \times 3}>0$. The conclusion that the signals $\widetilde{\delta}$ and $\tilde{\chi}$ are bounded can be drawn from (20) and Lemma 2. Also, the following equation can be obtained:

$$
\left|V_{\delta}(t)\right| \leq \frac{2 B}{B_{0}}
$$

Considering (19) and (22), it yields that

$$
\|\widetilde{\delta}(t)\| \leq \sqrt{\frac{4 B}{B_{0}}} .
$$

Therefore, the approximation error of the developed disturbance observer is bounded. This means that, for the subordinate system (9) and the fractional disturbance observer (14), the disturbance approximation error is bounded.

Remark 2. Based on the available information of the control object, such as system state, control input, or measurable variable, the unknown disturbance can be estimated by the disturbance observer which is used to design the controller. According to (14), it can be judged that the disturbance observer can be calculated by the appropriate adaptive law and control law.

3.2. Stability Analysis. In this part, a control scheme is proposed based on the design of disturbance observer and controller so that the fractional-order master system and the fractional-order slave system with disturbance are synchronized. Let the synchronization error be defined as

$$
e(t)=\xi(t)-x(t)
$$

where $e(t)=\left[e_{1}(t), e_{2}(t), e_{3}(t)\right]^{T}$.

Using (24) together with system (8) and system (9) yields

$$
\left\{\begin{array}{l}
\mathscr{D}_{t}^{v} e_{1}(t)=a e_{1}(t)+b x_{2}(t) e_{3}(t)+b e_{2}(t) \xi_{3}(t)+\delta_{1}(t)+u_{1}(t), \\
\mathscr{D}_{t}^{v} e_{2}(t)=c e_{2}(t)+x_{1}(t) e_{3}(t)+e_{1}(t) \xi_{3}(t)+\delta_{2}(t)+u_{2}(t), \\
\mathscr{D}_{t}^{v} e_{3}(t)=d e_{3}(t)+e_{2}(t)\left(e x_{1}(t)+x_{2}(t)+\xi_{2}(t)\right)+e e_{1}(t) \xi_{2}(t)+\delta_{3}(t)+u_{3}(t) .
\end{array}\right.
$$

Based on the disturbance observer, synchronization control inputs are designed as 


$$
\left\{\begin{array}{l}
u_{1}(t)=-a e_{1}(t)-b x_{2}(t) e_{3}(t)-b e_{2}(t) \xi_{3}(t)-\widehat{\delta}_{1}(t)-\mu_{1} e_{1}(t)-\widehat{k}_{1}(t) \operatorname{sign}\left(e_{1}(t)\right) \\
u_{2}(t)=-c e_{2}(t)-x_{1}(t) e_{3}(t)-e_{1}(t) \xi_{3}(t)-\widehat{\delta}_{2}(t)-\mu_{2} e_{2}(t)-\widehat{k}_{2}(t) \operatorname{sign}\left(e_{2}(t)\right) \\
u_{3}(t)=-d e_{3}(t)-e_{2}(t)\left(e x_{1}(t)+x_{2}(t)+\xi_{2}(t)\right)-e e_{1}(t) \xi_{2}(t)-\widehat{\delta}_{3}(t) \\
-\mu_{3} e_{3}(t)-\widehat{k}_{3}(t) \operatorname{sign}\left(e_{3}(t)\right),
\end{array}\right.
$$

where $\mu_{1}, \mu_{2}$, and $\mu_{3}$ are positive constants and $\widehat{k}_{i}$ is an estimate of $k_{i}, i=1,2,3$.

The adaptation laws of the design estimate $\widehat{k}_{i}$ is written as

$$
\mathscr{D}_{t}^{v} \widehat{k}_{i}=\eta_{i}\left(\left|e_{i}(t)\right|-\widehat{k}_{i}\right) \text {, }
$$

where $\eta_{i}$ is a positive constant.

According to the above discussion, we need to verify the rationality and effectiveness of the control plan.

Theorem 2. Under the observer (14), the controller (26), and the estimation of the parameters (27), the synchronization error (24) of system (8) and system (9) will converge to a small compact set.

Proof. Let a Lyapunov function candidate be given by

$$
V_{o}(t)=\frac{1}{2} e^{T}(t) e(t)+\frac{1}{2} \widetilde{\delta}^{T}(t) \widetilde{\delta}(t)+\frac{1}{2} \sum_{i=1}^{3} \frac{1}{\eta_{i}} \widetilde{k}_{i}^{2},
$$
where $\tilde{k}_{i}=k_{i}-\widehat{k}_{i}$ and $k_{i}$ is a constant.

Invoking Lemma 2 , the fractional derivative of $V_{o}(t)$ along the state trajectory is

$$
\mathscr{D}_{t}^{v} V_{o}(t) \leq e^{T}(t) \mathscr{D}_{t}^{v} e(t)+\widetilde{\delta}^{T}(t) \mathscr{D}_{t}^{v} \widetilde{\delta}(t)+\sum_{i=1}^{3} \frac{1}{\eta_{i}} \widetilde{k}_{i} \mathscr{D}_{t}^{v} \widetilde{k}_{i}
$$

Combining (25), (26), and (29) and employing Property 1 , the following can be inferred:

$$
\begin{aligned}
\mathscr{D}_{t}^{\nu} V_{o}(t) & \leq \sum_{i=1}^{3} e_{i}(t)\left[\widetilde{\delta}_{i}(t)-\mu_{i} e_{i}(t)-\widehat{k}_{i}(t) \operatorname{sign}\left(e_{i}(t)\right)\right]+\widetilde{\delta}^{T}(t) \mathscr{D}_{t}^{v} \widetilde{\delta}(t)-\sum_{i=1}^{3} \frac{1}{\eta_{i}} \widetilde{k}_{i} \mathscr{D}_{t}^{v} \widehat{k}_{i} \\
& =\sum_{i=1}^{3} e_{i}(t)\left[\widetilde{\delta}_{i}(t)-\mu_{i} e_{i}(t)\right]-\sum_{i=1}^{3}\left[\left|e_{i}(t)\right| \widehat{k}_{i}+\frac{1}{\eta_{i}} \widetilde{k}_{i} \mathscr{D}_{t}^{v} \widehat{k}_{i}\right]+\widetilde{\delta}^{T}(t) \mathscr{D}_{t}^{v} \widetilde{\delta}(t) \\
& =-\sum_{i=1}^{3} \mu_{i} e_{i}^{2}(t)+\sum_{i=1}^{3} e_{i}(t) \widetilde{\delta}_{i}(t)-\sum_{i=1}^{3}\left[\left|e_{i}(t)\right|\left(k_{i}-\widetilde{k}_{i}\right)+\frac{1}{\eta_{i}} \widetilde{k}_{i} \mathscr{D}_{t}^{v} \widehat{k}_{i}\right]+\widetilde{\delta}^{T}(t) \mathscr{D}_{t}^{v} \widetilde{\delta}(t) \\
& \leq-\sum_{i=1}^{3} \mu_{i} e_{i}^{2}(t)+\sum_{i=1}^{3} e_{i}(t) \widetilde{\delta}_{i}(t)+\sum_{i=1}^{3}\left[\left|e_{i}(t)\right| \widetilde{k}_{i}-\frac{1}{\eta_{i}} \widetilde{k}_{i} \mathscr{D}_{t}^{v} \widehat{k}_{i}\right]+\widetilde{\delta}^{T}(t) \mathscr{D}_{t}^{v} \widetilde{\delta}(t) .
\end{aligned}
$$

According to the Young's inequality and the parameter adaptation law (27) that has been designed, it can be obtained that

$$
\begin{aligned}
\mathscr{D}_{t}^{\nu} V_{o}(t) & \leq-\sum_{i=1}^{3} \mu_{i} e_{i}^{2}(t)+\sum_{i=1}^{3} \frac{1}{2} e_{i}^{2}(t)+\sum_{i=1}^{3} \frac{1}{2} \widetilde{\delta}_{i}^{2}(t)+\sum_{i=1}^{3} \widetilde{k}_{i} \widehat{k}_{i}+\widetilde{\delta}^{T}(t) \mathscr{D}_{t}^{\nu} \widetilde{\delta}(t) \\
& =-\sum_{i=1}^{3}\left(\mu_{i}-0.5\right) e_{i}^{2}(t)+\sum_{i=1}^{3} \frac{1}{2} \widetilde{\delta}_{i}^{2}(t)+\sum_{i=1}^{3} \widetilde{k}_{i}\left(k_{i}-\widetilde{k}_{i}\right)+\widetilde{\delta}^{T}(t) \mathscr{D}_{t}^{\nu} \widetilde{\delta}(t) \\
& \leq-\sum_{i=1}^{3}\left(\mu_{i}-0.5\right) e_{i}^{2}(t)+\sum_{i=1}^{3} \frac{1}{2} \widetilde{\delta}_{i}^{2}(t)-\sum_{i=1}^{3} \frac{1}{2} \widetilde{k}_{i}^{2}+\sum_{i=1}^{3} \frac{1}{2} k_{i}^{2}+\widetilde{\delta}^{T}(t) \mathscr{D}_{t}^{\nu} \widetilde{\delta}(t) .
\end{aligned}
$$

Utilizing (20) and (30) leads to 


$$
\begin{aligned}
\mathscr{D}_{t}^{\nu} V_{o}(t) \leq & -\sum_{i=1}^{3}\left(\mu_{i}-0.5\right) e_{i}^{2}(t)+\sum_{i=1}^{3} \frac{1}{2} \widetilde{\delta}_{i}^{2}(t)-\sum_{i=1}^{3} \frac{1}{2} \widetilde{k}_{i}^{2}+\sum_{i=1}^{3} \frac{1}{2} k_{i}^{2}+\widetilde{\delta}^{T}(t) \mathscr{D}_{t}^{\nu} \widetilde{\delta}(t) \\
\leq & -\sum_{i=1}^{3}\left(\mu_{i}-0.5\right) e_{i}^{2}(t)+\sum_{i=1}^{3} \frac{1}{2} \widetilde{\delta}_{i}^{2}(t)-\sum_{i=1}^{3} \frac{1}{2} \widetilde{k}_{i}^{2}+\sum_{i=1}^{3} \frac{1}{2} k_{i}^{2}-\widetilde{\delta}^{T}(t)\left(\rho-0.5 I_{3 \times 3}\right) \widetilde{\delta}(t)+\frac{1}{2} \bar{\delta}^{2} \\
= & -\sum_{i=1}^{3}\left(\mu_{i}-0.5\right) e_{i}^{2}(t)-\sum_{i=1}^{3}\left(\rho_{i}-1\right) \widetilde{\delta}_{i}^{2}(t)-\sum_{i=1}^{3} \frac{1}{2} \widetilde{k}_{i}^{2}+\sum_{i=1}^{3} \frac{1}{2} k_{i}^{2}+\frac{1}{2} \bar{\delta}^{2} \\
= & -\frac{1}{2} e^{T}(t) 2\left(\mu-0.5 I_{3 \times 3}\right) e(t) \\
& -\frac{1}{2} \widetilde{\delta}^{T}(t) 2\left(\rho-I_{3 \times 3}\right) \widetilde{\delta}(t)-\sum_{i=1}^{3} \eta_{i} \frac{1}{2 \eta_{i}} \widetilde{k}_{i}^{2}+\sum_{i=1}^{3}\left(\frac{1}{2} k_{i}^{2}+\frac{1}{2} \bar{\delta}_{i}^{2}\right) \\
\leq & -\kappa V_{o}(t)+\theta,
\end{aligned}
$$

where $\rho=\operatorname{diag}\left(\rho_{1}, \rho_{2}, \rho_{3}\right), \mu=\operatorname{diag}\left(\mu_{1}, \mu_{2}, \mu_{3}\right)$ are diagonal matrices, and $\kappa$ and $\theta$ are defined by

$$
\begin{aligned}
\kappa & :=\min \left\{\lambda_{\min }\left(2 \mu-I_{3 \times 3}\right), \lambda_{\min }\left(2 \rho-2 I_{3 \times 3}\right), \eta_{i}\right\}, \\
\theta & :=\sum_{i=1}^{3}\left(\frac{1}{2} k_{i}^{2}+\frac{1}{2} \bar{\delta}_{i}^{2}\right) .
\end{aligned}
$$

To ensure the stability of the closed-loop system, the corresponding design parameters $\eta_{i}$ and $\mu$ and $\rho$ should be chosen to satisfy

$$
\begin{aligned}
\eta_{i} & >0, \\
2 \mu-I_{3 \times 3} & >0, \\
2 \rho-2 I_{3 \times 3} & >0 .
\end{aligned}
$$

According to (32) and Lemma 2, it yields that

$$
V_{o}(t) \leq \frac{2 \theta}{\kappa} .
$$

Therefore, it may directly represent that the signals $e(t)$, $\widetilde{\delta}(t)$, and $\widetilde{k}_{i}$ are bounded, and the synchronization errors converge to any small neighborhood of the origin. In other words, the synchronization between system (8) and subordinate system (9) can be achieved.

Remark 3. The proposed fractional adaptive controller of (26) and (27) is applicable for synchronization of two identical uncertain fractional-order chaotic systems with different initial conditions. It should be noticed that the introduction of $\operatorname{sign}(e(t))$ is convenient for stability analysis, and its coefficient $k_{i}$ is a very small and unknown constant that can be estimated by $\widehat{k}_{i}$ and (27) in the design of the controller.

\section{Simulation Example}

In this section, the effectiveness of the proposed control scheme and the feasibility of the fractional disturbance observer design are illustrated by computational simulations of chaotic system (8) and subordinate system (9). The numerical simulation uses MATLAB software to give the approximation effect of the disturbance observer, the synchronization performance of the two systems and the control performance of the controller will be specifically analyzed in the following.

In this example, the parameters of the chaotic master system (8) and the subordinate system (9) are selected as $a=-1, b=-10, c=1, d=-0.78$, and $e=-8$. When the fractional order of the system is taken as $v=0.96$ and the initial value of the vector of the chaotic system are taken as $[2,1,1]$ and $[5,1,1]$, two two-wing chaotic attractors and two fourwing chaotic attractors can be obtained as shown in Figure 1.

The balance point of system (8) can be calculated, as shown in Table 1.

Linearize system (8) at the equilibrium points $\left(\hat{x}_{1}, \hat{x}_{2}, \hat{x}_{3}\right)$ to get the matrix

$$
A=\left(\begin{array}{ccc}
-2 & -10 \widehat{x}_{3} & -10 \widehat{x}_{2} \\
\widehat{x}_{3} & 1 & \widehat{x}_{1} \\
-8 \widehat{x}_{2} & 2 \widehat{x}_{2}-8 \widehat{x}_{1} & -0.78
\end{array}\right) .
$$

The characteristic polynomial of the matrix is written as $f(\lambda)=\lambda^{3}+A_{2} \lambda^{2}+A_{1} \lambda+A_{0}$, where $A_{2}=1.78, A_{1}=8 \widehat{x}_{1}^{2}+$ $10 \widehat{x}_{3}^{2}-80 \widehat{x}_{2}^{2}-2 \widehat{x}_{1} \widehat{x}_{2}-1.22$ and $A_{0}=16 \widehat{x}_{1}^{2}+80 \widehat{x}_{2}^{2}+7.2 \widehat{x}_{3}^{2}+$ $20 \widehat{x}_{2}^{2} \widehat{x}_{3}-4 \widehat{x}_{1} \hat{x}_{2}-160 \widehat{x}_{1} \hat{x}_{2} \hat{x}_{3}-1.56$.

According to Routh-Hurwitz method, the equilibrium point is stable if and only if $A_{2}>0, A_{0}>0$, and $A_{2} A_{1}-A_{0}>0$. The eigenvalues and stability corresponding to the equilibrium point are shown in Table 2 .

According to the calculation results in Table 2, the chaotic attractors are generated around $S_{2}, S_{3}, S_{4}, S_{5}$, and $S_{1}$ acting as a connecting ring. In order to make the chaotic attractors exist in the fractional-order system, it is necessary to ensure that the eigenvalue corresponding to $S_{2}, S_{3}, S_{4}$, and $S_{5}$ are unstable, and then the following must be satisfied [26].

$$
|\arg (\lambda)| \leq \frac{1}{2} \pi \nu .
$$




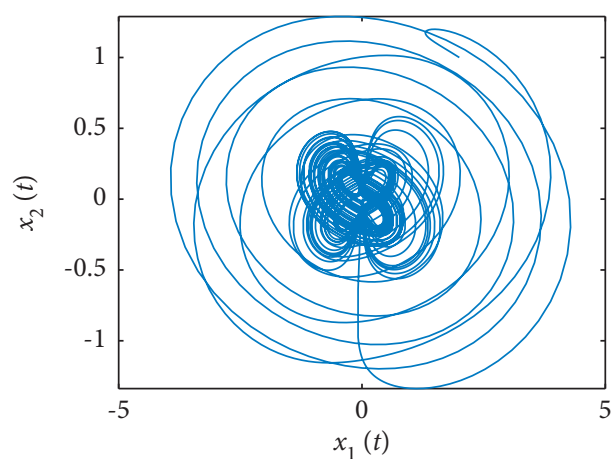

$[2,1,1]$

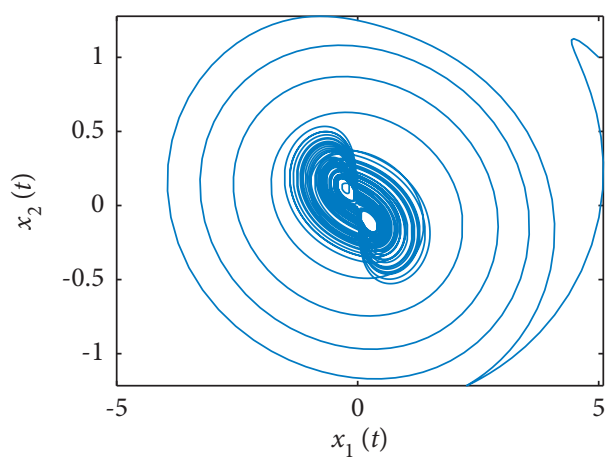

$-[5,1,1]$

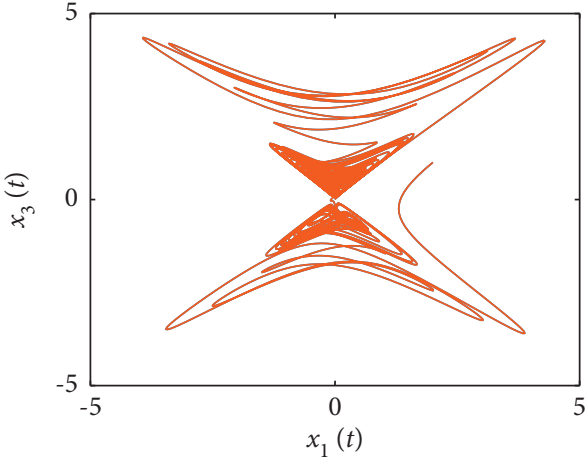

$[2,1,1]$

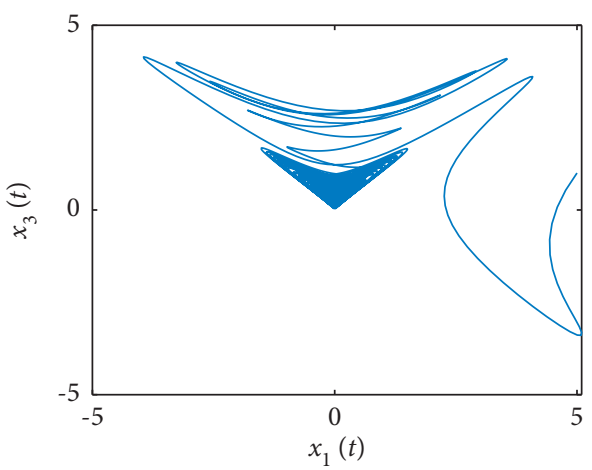

$[-[5,1,1]$

FIgURE 1: Chaotic behavior of fractional-order $v=0.96$ with initial conditions $[2,1,1]$ and $[5,1,1]$.

TABLE 1: Equilibrium point of the system.

\begin{tabular}{lccc}
\hline$S$ & $x$ & $y$ & $z$ \\
\hline$S_{1}$ & 0 & 0 & 0 \\
$S_{2}$ & -0.30387 & 0.1359 & 0.4472 \\
$S_{3}$ & 0.30387 & -0.1359 & 0.4472 \\
$S_{4}$ & -0.32136 & -0.1437 & -0.4472 \\
$S_{5}$ & 0.32136 & 0.1437 & -0.4472 \\
\hline
\end{tabular}

TABLE 2: Stability of equilibrium points of systems.

\begin{tabular}{lcc}
\hline Equilibrium & Eigenvalue & Stability \\
\hline$S_{1}$ & $\lambda_{1}=-2, \lambda_{2}=-0.78, \lambda_{3}=1$ & Unstable \\
$S_{2}$ & $\lambda_{1}=-2.636, \lambda_{2}=0.428-1.49 i, \lambda_{3}=0.428+1.49 i$ & Unstable \\
$S_{3}$ & $\lambda_{1}=-2.636, \lambda_{2}=0.428-1.49 i, \lambda_{3}=0.428+1.49 i$ & Unstable \\
$S_{4}$ & $\lambda_{1}=-2.692, \lambda_{2}=0.456-1.452 i, \lambda_{3}=0.456+1.452 i$ & Unstable \\
$S_{5}$ & $\lambda_{1}=-2.692, \lambda_{2}=0.456-1.452 i, \lambda_{3}=0.456+1.452 i$ & Unstable \\
\hline
\end{tabular}

After calculating, the necessary condition for the existence of the chaotic attractors of the fractional-order chaotic system is $v>0.8224$.

The initial value vector of the fractional subordinate system (9) is written as $[4,3,2]$. The initial value of adaptive parameters, the intermediate variables to calculate the disturbance, and the external disturbances are, respectively, assumed to be $\widehat{k}=[0.1,0.1,0.1], \chi=[0.5,0.5,0.5]$, and $\widehat{\delta}=[0.1,0.9,0.1]$. The parameters of the disturbance observer are chosen as $\rho=[10,10,10]$, and the parameters of the system controller are specified as $\mu=[20,20,20]$ and $\eta=[0.1,0.1,0.1]$. The external disturbance can be assumed as $\delta=[\sin 2 t, \cos 2 t, \sin 2 t]$. Figures 2 to 4 show that, under the control method mentioned in this article, the subordinate system (9) reaches synchronization with the master system (8) at $t=0.6$. The value of $\widehat{k}_{i}$ in Figure 5 decreases monotonically and tends to 0 , which responds to the description in Remark 3. Figure 6 shows that the controllers converge to the origin in a small area within a limited time, reflecting the efficiency and practicability of the control 


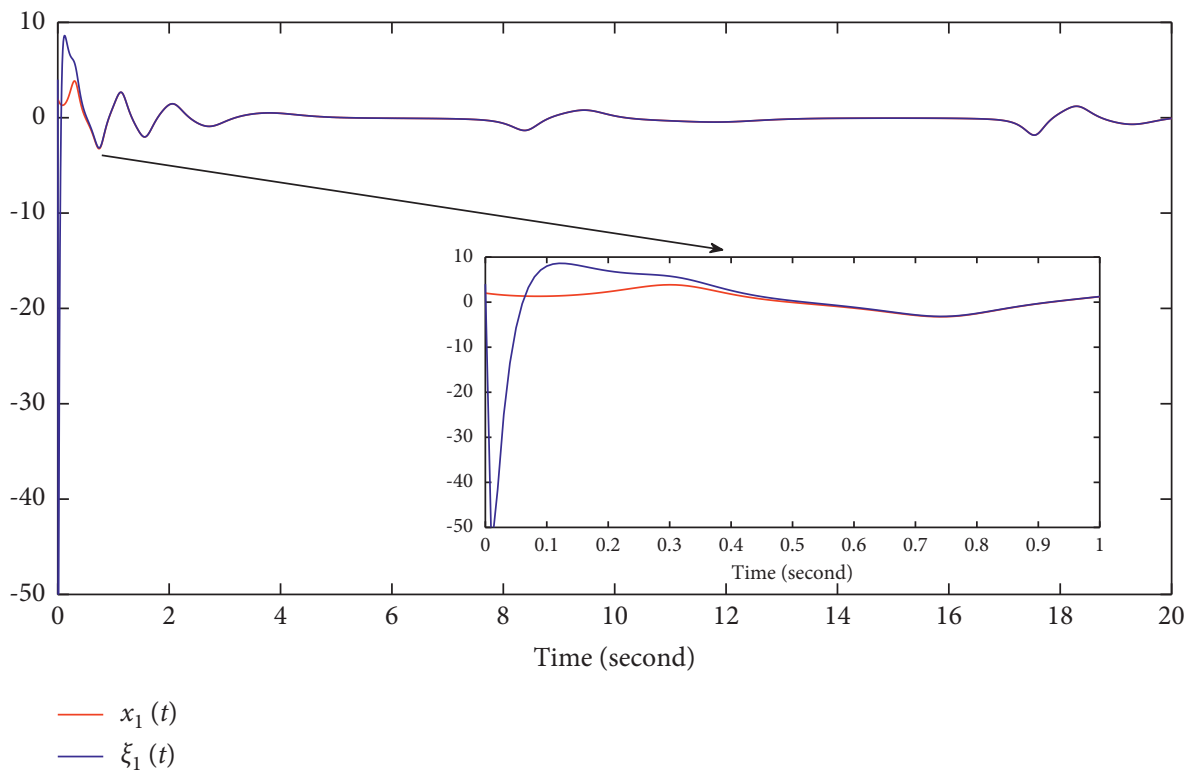

Figure 2: $x_{1}$ and $y_{1}$.

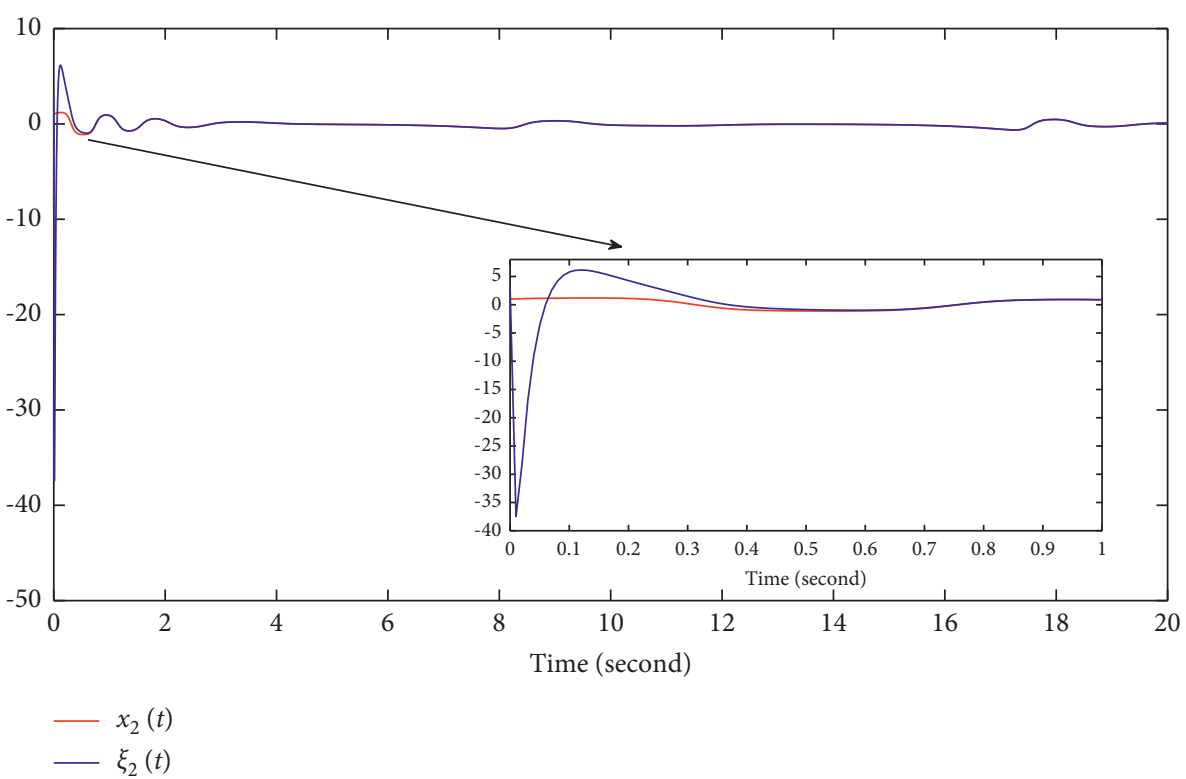

Figure 3: $x_{2}$ and $y_{2}$.

method. The efficient fitting synchronization of the two systems is shown in Figure 7. Finally, it can be seen that the disturbance observers effectively monitor the external disturbance, which indicate that the disturbance observers perform well in Figure 8. The above results show that the synchronization control scheme and disturbance observer design method proposed in this paper can effectively carry out tracking control. 


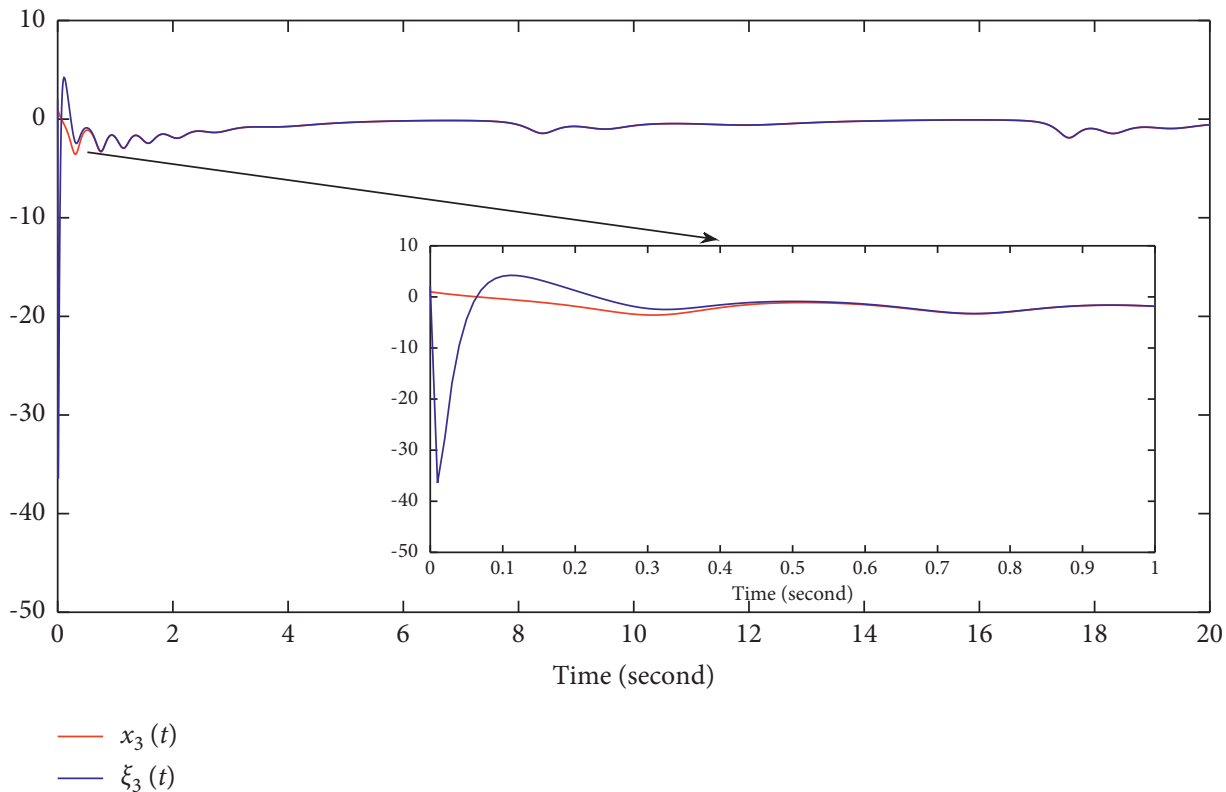

FiguRe 4: $x_{3} y_{3}$.

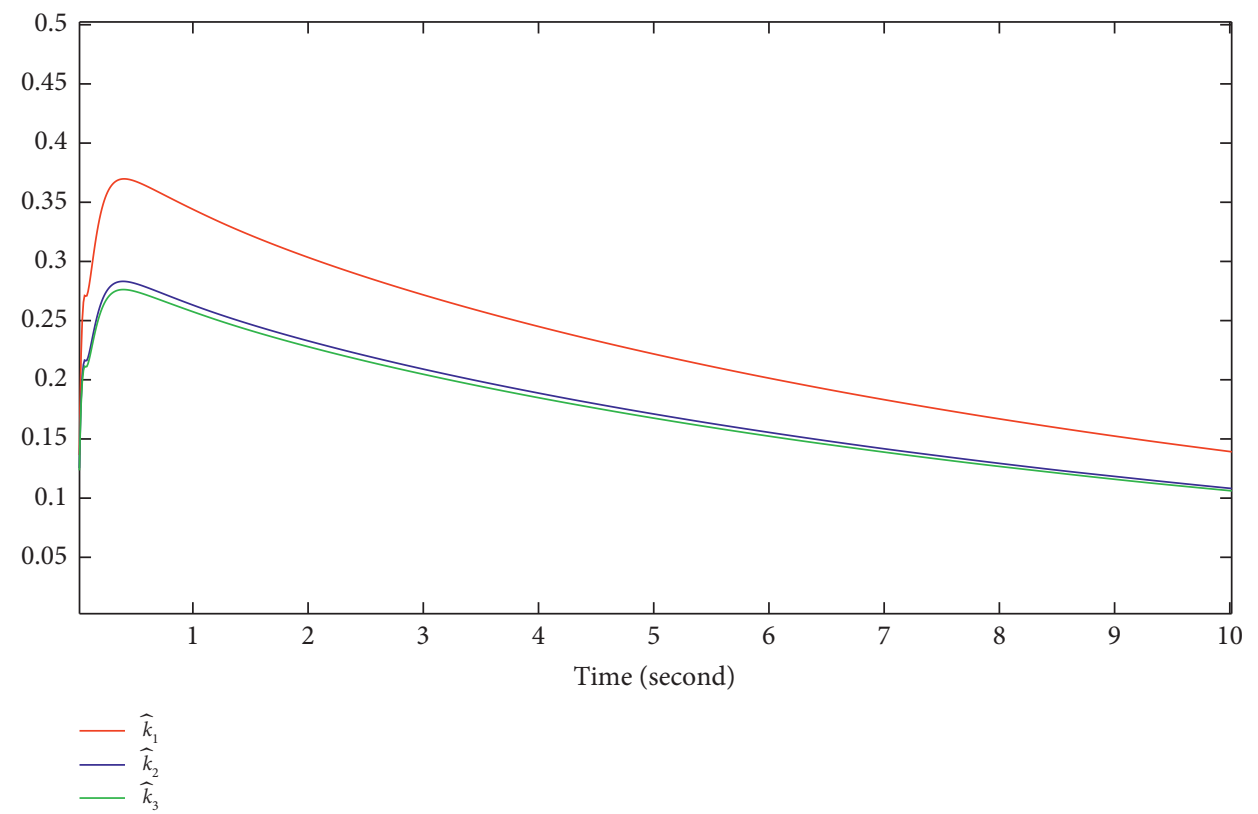

FIGURE 5: Estimated parameters $\widehat{k}_{1}, \widehat{k}_{2}$, and $\widehat{k}_{3}$. 

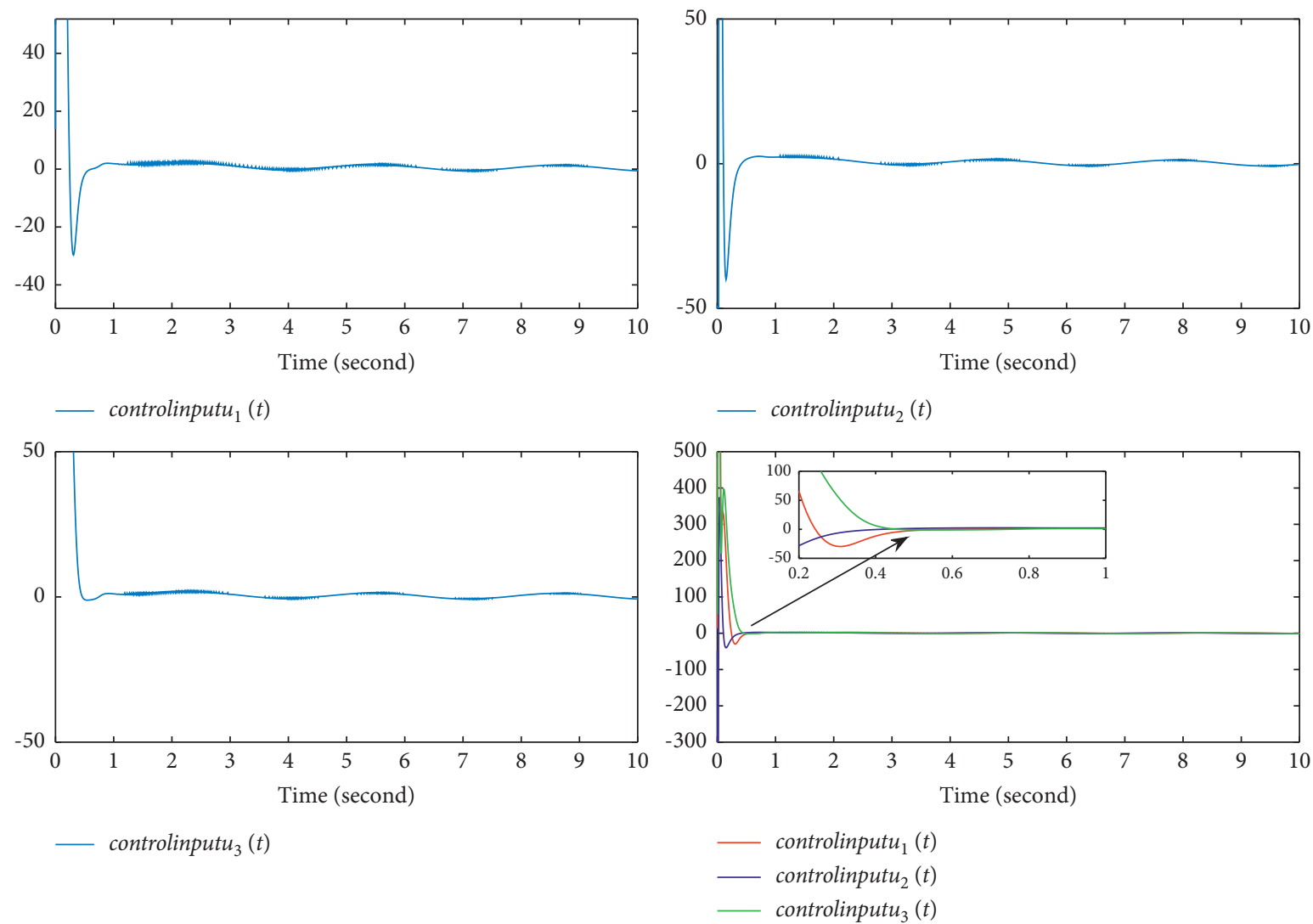

FIGURE 6: Control inputs $u_{1}, u_{2}$, and $u_{3}$.
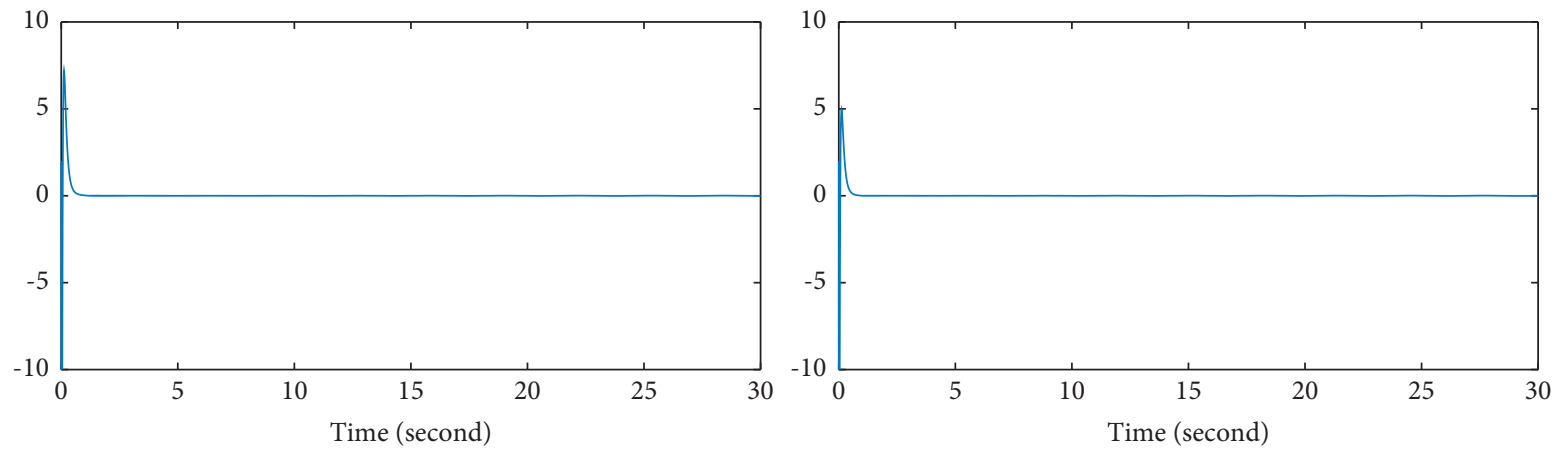

$$
\text { - } \text { errore }_{1}(t)
$$

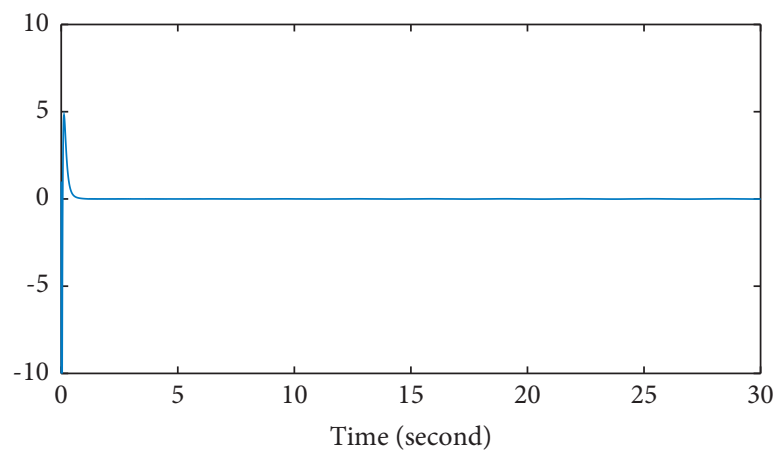

- errore $_{3}(t)$
- errore $_{2}(t)$

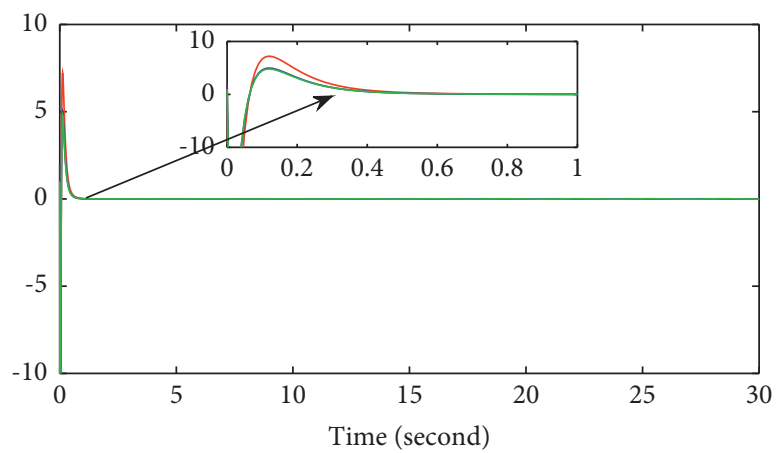

- errore $_{1}(t)$
- errore $_{2}(t)$
- errore $_{3}(t)$

FIGURE 7: Synchronization errors $e_{1}, e_{2}$, and $e_{3}$. 

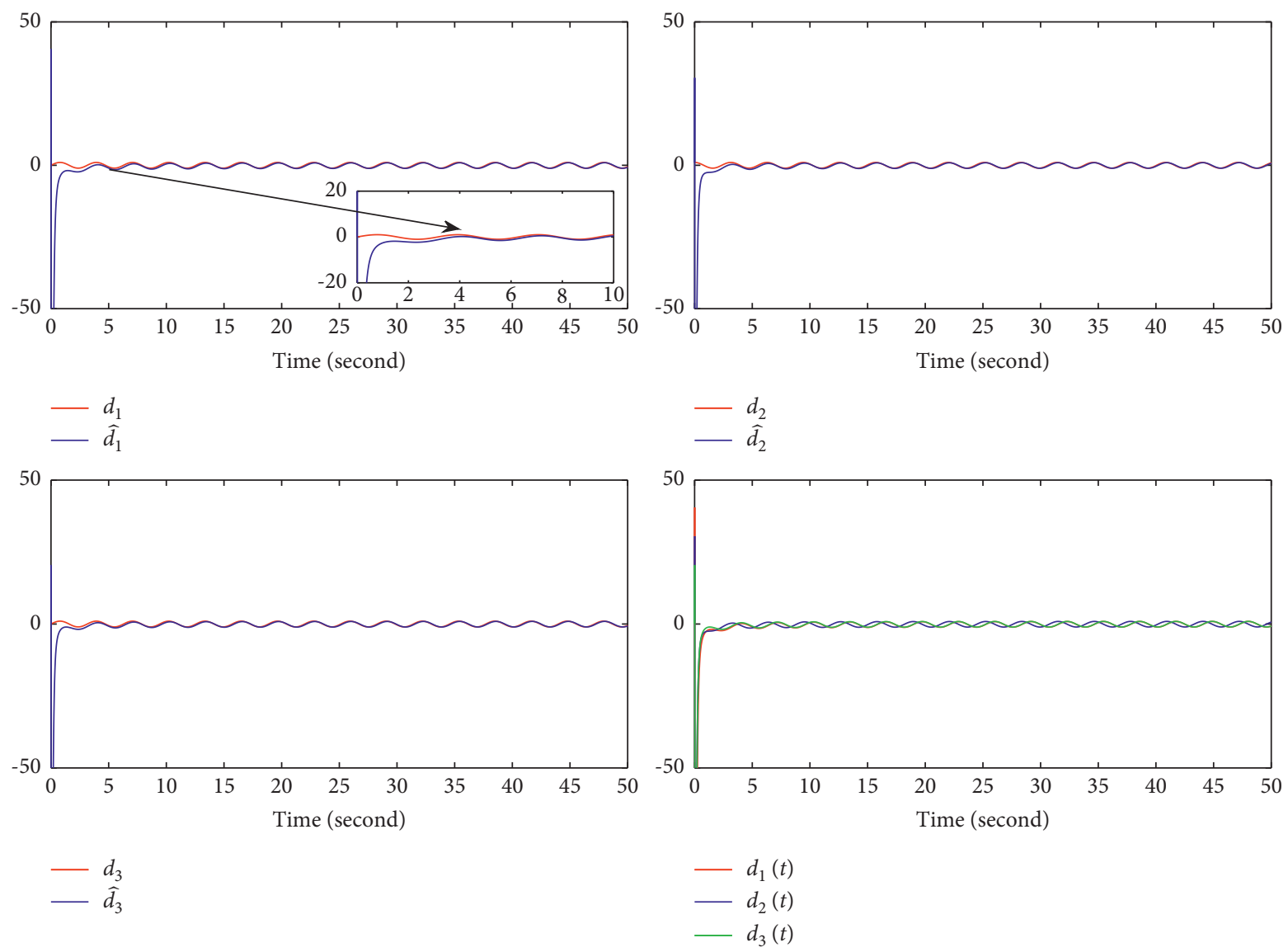

FIGURE 8: Disturbance observer.

\section{Conclusions}

In this paper, a class of subordinate system is designed to track synchronization and stability analysis of the fractionalorder chaotic system. The control scheme is designed using the adaptive law of unknown parameters, so that the system can achieve synchronous control in limited time. Then, based on the stability theory of Lyapunov function, the stability of the closed-loop system is proved. Numerical simulation experiments show that the control scheme designed in this paper is feasible for the fractional-order chaotic system. It is worth noting that the design of disturbance observer can be used in various nonlinear systems with disturbances and uncertainties. Although the proposed method has good control effect, the robustness is not satisfactory. How to solve this problem is our future research direction.

\section{Data Availability}

No data were used to support the findings of this study.

\section{Conflicts of Interest}

The authors declare that there are no conflicts of interest related to this article.

\section{References}

[1] H. Liu, S.-G. Li, Y.-G. Sun, and H.-X. Wang, "Prescribed performance synchronization for fractional-order chaotic systems," Chinese Physics B, vol. 24, no. 9, pp. 153-160, 2015.

[2] Y. Wei, Y. Chen, S. Liang, and Y. Wang, "A novel algorithm on adaptive back stepping control of fractional order systems," Neurocomputing, vol. 165, pp. 395-402, 2015.

[3] D. Sheng, Y. Wei, S. Cheng, and Y. Wang, "Observer-based adaptive back stepping control for fractional order systems with input saturation," ISA Transactions, vol. 82, pp. 18-29, 2018.

[4] C. Wu and X. Liu, "Lyapunov and external stability of caputo fractional order switching systems," Nonlinear Analysis: Hybrid Systems, vol. 34, pp. 131-146, 2019.

[5] H. Liu, S. Li, G. Li, and H. Wang, "Robust adaptive control for fractional-order financial chaotic systems with system uncertainties and external disturbances," Information Technology and Control, vol. 46, no. 2, pp. 246-259, 2017.

[6] R. Cui, Y. Wei, S. Cheng, and Y. Wang, "An innovative parameter estimation for fractional order systems with impulse noise," ISA Transactions, vol. 82, pp. 120-129, 2018.

[7] S.-Y. Shao, M. Chen, and Q.-X. Wu, "Stabilization control of continuous-time fractional positive systems based on disturbance observer," IEEE Access, vol. 4, pp. 3054-3064, 2016.

[8] A. J. Muñoz-Vázquez, M. B. Ortiz-Moctezuma, A. SánchezOrta, and V. Parra-Vega, "Adaptive robust control of fractional-order systems with matched and mismatched 
disturbances," Mathematics and Computers in Simulation, vol. 162, pp. 85-96, 2019.

[9] T. Zhou, Z. Zuo, and Y. Wang, "Quantizer-based triggered control for chaotic synchronization with information constraints," IEEE transactions on cybernetics, vol. 48, no. 8, pp. 2500-2508, 2017.

[10] J. Chen, C. Li, and X. Yang, "Chaos synchronization of the distributed-order lorenz system via active control and applications in chaotic masking," International Journal of Bifurcation and Chaos, vol. 28, no. 10, pp. 1850121-1850882, 2018.

[11] A. S. Al-Obeidi and S. F. Al-Azzawi, "Complete synchronization of a novel 6-d hyperchaotic lorenz system with known parameters," International Journal of Engineering \& Technology, vol. 7, no. 4, pp. 5345-5349, 2018.

[12] D. Nikitin, I. Omelchenko, A. Zakharova, M. Avetyan, A. L. Fradkov, and E. Schöll, "Complex partial synchronization patterns in networks of delay-coupled neurons," Philosophical Transactions of the Royal Society A, vol. 377, no. 2153, Article ID 20180128, 2019.

[13] D. Ding, X. Qian, N. Wang, and D. Liang, "Synchronization and anti-synchronization of a fractional order delayed memristor-based chaotic system using active control," Modern Physics Letters B, vol. 32, no. 14, Article ID 1850142, 2018.

[14] A. Ouannas, A. T. Azar, T. Ziar, and A. G. Radwan, "Generalized synchronization of different dimensional integerorder and fractional order chaotic systems," in Studies in Computational Intelligence, vol. 688, pp. 671-697, Springer, Cham, Switzerland, 2017.

[15] A. Fan and J. Li, "Adaptive neural network prescribed performance matrix projection synchronization for unknown complex dynamical networks with different dimensions," Neurocomputing, vol. 281, pp. 55-66, 2018.

[16] S. Mobayen and J. Ma, "Robust finite-time composite nonlinear feedback control for synchronization of uncertain chaotic systems with nonlinearity and time-delay," Chaos, Solitons \& Fractals, vol. 114, pp. 46-54, 2018.

[17] O. Mofid, M. Momeni, S. Mobayen, and A. Fekih, "A disturbance-observer-based sliding mode control for the robust synchronization of uncertain delayed chaotic systems: application to data security," IEEE Access, vol. 9, pp. 1654616555, 2021.

[18] Y. Mousavi and A. Alfi, "Fractional calculus-based firefly algorithm applied to parameter estimation of chaotic systems," Chaos, Solitons \& Fractals, vol. 114, pp. 202-215, 2018.

[19] B. Wu, M. Chen, and L. Zhang, "Disturbance-observer-based sliding mode control for T-S fuzzy discrete-time systems with application to circuit system," Fuzzy Sets and Systems, vol. 374 , pp. 138-151, 2019.

[20] A. Merabet, M. Ouhrouche, and R. Bui, "Nonlinear predictive control with disturbance observer for induction motor drive," in Proceedings of the IEEE International Symposium on Industrial Electronics, pp. 86-91, IEEE, Montreal, QC, Canada, July 2006.

[21] S. Chen, Z. Chen, and Z. Zhao, "Parameter selection and performance analysis of linear disturbance observer based control for a class of nonlinear uncertain systems," IEEE Transactions on Industrial Electronics, 2021.

[22] M. Teodor, S. Pilipovic, B. Stankovic, and D. Zorica, Fractional Calculus with Applications in Mechanics: Vibrations and Diffusion Processes, John Wiley \& Sons, 2014.

[23] N. Aguila-Camacho, M. A. Duarte-Mermoud, and J. A. Gallegos, "Lyapunov functions for fractional order systems," Communications in Nonlinear Science and $\mathrm{Nu}$ merical Simulation, vol. 19, no. 9, pp. 2951-2957, 2014.

[24] K. Diethelm and N. J. Ford, "Analysis of fractional differential equations," Journal of Mathematical Analysis and Applications, vol. 265, no. 2, pp. 229-248, 2002.

[25] M. A. Duarte-Mermoud, N. Aguila-Camacho, J. A. Gallegos, and R. Castro-Linares, "Using general quadratic lyapunov functions to prove lyapunov uniform stability for fractional order systems," Communications in Nonlinear Science and Numerical Simulation, vol. 22, no. 1-3, pp. 650-659, 2015.

[26] M. Saleh Tavazoei and M. Haeri, "A necessary condition for double scroll attractor existence in fractional-order systems," Physics Letters A, vol. 367, no. 1-2, pp. 102-113, 2007. 